Vol. 45 (1992) [525-526]

\title{
On triangles with rational altitudes, angle bisectors or medians
}

\author{
RALPH HEINER BUChHOLZ
}

This thesis is principally concerned with integer-sided triangles. Such a triangle is called a Heron triangle if its area is also an integer. The main theme of the thesis is a study of the relationships between Heron triangles and triangles with various types of cevians of integer length. Cevians are lines joining the vertices of a triangle to any points on the opposite sides.

The first chapter covers the case of three integer altitudes and initially considers isosceles and Pythagorean examples. We then examine two transformations, the hinge and the pivot, which can be used to classify integer-sided triangles with three integer altitudes. The general parametrisation emerges from an analogous one for the set of Heron triangles.

The second chapter deals with the case of three angle bisectors of integer length. Again we search for isosceles and Pythagorean examples but this time it turns out that Pythagorean triangles with three integer angle bisectors do not exist. Next we show that, somewhat surprisingly, any integer-sided triangle with three integer angle bisectors must necessarily have integer area. This leads to a general parametrisation of such triangles, by the same technique as was used in Chapter 1.

The third chapter deals with the case of three integer medians. Previously known results are that the semiperimeter must be even and that the primitive triangles occur in related pairs. The search for simple examples leads to negative results, for example, such triangles cannot be isosceles, Pythagorean or have sides in an arithmetic progression.

One of the unsolved problems in this area asks whether there is a nonempty intersection between the set of Heron triangles and the set of triangles with three integer medians. To this end we note that Euler's parametrisation of a proper subset of triangles with three integer medians does not include any triangle with integer area.

\footnotetext{
Received 22nd October, 1991.
}

Thesis submitted to the University of Newcastle, November 1989. Degree approved May 1991. Supervisor: Associate Professor Roger B. Eggleton.

Copyright Clearance Centre, Inc. Serial-fee code: 0004-9729/92 \$A2.00+0.00. 
A complete parametrisation is given for triangles with three rational sides and two rational medians. This leads to a method for generating triangles with three rational medians by application of the tangent-chord process to various elliptic curves.

In the fourth chapter we convert the parametrisation of Chapter 3 into a computer search routine. This leads to the discovery of several examples of Heron triangles with two integer medians. These all lie on a surface defined by a polynomial function of two variables. The problem of discovering rational points on this surface turns out to be difficult to attack with any known techniques.

The fifth and final chapter deals with rational concurrent cevians through arbitrary points inside and outside a triangle. The defining equations are considered along with some interesting special cases, for example, cevians through the circumcentre. The relationship between three rational cevians and a rational tiling of the triangle is also discussed.

\section{CMR Group}

H-Block Victorial Barracks

St. Kilda Rd

Melbourne Vic 3000

Australia 